\title{
ORIGINAL ARTICLE Optimization of lentiviral vector production for scale-up in fixed-bed bioreactor
}

\author{
AJ Valkama ${ }^{1,2,3,6}$, HM Leinonen ${ }^{2,3,6}$, EM Lipponen ${ }^{2,3,4}$, V Turkki ${ }^{1,2,3}$, J Malinen ${ }^{1,2,3}$, T Heikura ${ }^{1}$, S Ylä-Herttuala ${ }^{1,5}$ and HP Lesch ${ }^{1,2,3,4}$
}

Lentiviral vectors (LVs) are promising tools for gene therapy. However, scaling up the production methods of LVs in order to produce high-quality vectors for clinical purposes has proven to be difficult. In this article, we present a scalable and efficient method to produce LVs with transient transfection of adherent 293T cells in a fixed-bed bioreactor. The disposable iCELLis bioreactors are scalable with a large three-dimensional (3D) growth area range between 0.53 and $500 \mathrm{~m}^{2}$, an integrated perfusion system, and a controllable environment for production. In this study, iCELLis Nano $\left(2.67-4 \mathrm{~m}^{2}\right)$ was used for optimizing production parameters for scale-up. Transfections were first done using traditional calcium phosphate method, but in later runs polyethylenimine was found to be more reliable and easier to use. For scalable LV production, perfusion rate control by measuring cell metabolite concentrations in the bioreactor leads to higher productivity and reduced costs. Optimization of cell seeding density for targeted cell concentration during transfection, use of low compaction fixed-bed and lowering the culture $\mathrm{pH}$ have a positive effect on LV productivity. These results show for the first time that iCELLis bioreactor is scalable from bench level to clinical scale LV production.

Gene Therapy (2018) 25, 39-46; doi:10.1038/gt.2017.91

\section{INTRODUCTION}

Lentiviral vectors (LVs) have emerged as promising vector types and potentially a safer alternative to $\gamma$-retroviral vectors. Utilization of LVs in clinical trials has increased from $2.9 \%$ in 2012 to $6.3 \%$ in 2017. ${ }^{1,2}$ LVs are especially applicable to gene therapy. They can generally infect both dividing and non-dividing cells, ${ }^{3}$ and efficiently transduce target cells inducing a long-term transgene expression. ${ }^{4,5}$ Moreover, LVs have not demonstrated the oncogenic features of $\gamma$-retroviral vectors encountered in clinical trials, namely integration site preference. ${ }^{6,7}$

However, LV manufacturing methods require upgrading to meet the current demands. Early phase clinical trials require extensive amounts of $\mathrm{LVs}^{8}{ }^{8}$ which are still mostly produced by non-standardized, expensive and labor-intensive two-dimensional (2D) systems. Bioreactors allow large-scale vector production in suspension or adherent 3D matrices, and are generally less labor intensive than conventional 2D systems.

Viral vector production is performed by transient transfection, packaging cell lines or transduction. Human embryonic kidney 293 cells are widely used for viral vector production because of high transfectability and adaptability. ${ }^{9}$ The human embryonic kidney 293 variant $293 T^{10,11}$ is especially efficient in high-titer LV production, $^{12-14}$ and it can adapt to both adherent and suspension growth. Although large-scale LV production can be executed in suspension conditions, ${ }^{9}$ adherent production is generally favored because of relatively high cell densities and thus higher production yields. ${ }^{15,16}$ LVs are typically produced with transient transfection rather than packaging cell lines. ${ }^{13,17}$ Most commonly applied methods include calcium phosphate (CaPho) precipitation and polyethylenimine (PEI). ${ }^{17,18}$
The PALL iCELLis is a compact fixed-bed bioreactor with an integrated perfusion system. iCELLis Nano provides up to $4 \mathrm{~m}^{2}$ cell culture area, and iCELLis 500 scales up to $500 \mathrm{~m}^{2}$, corresponding to growth area of approximately 800 CF10 (Cell Factory) 2D culture vessels. ${ }^{19,20}$ iCELLis allows scaling of adherent production in a controlled environment, and the highly integrated single-use equipment can be adapted to meet current good manufacturing practices requirements. Viral vaccines, ${ }^{21}$ recombinant proteins, ${ }^{22}$ adeno-associated viral vectors ${ }^{23}$ and retroviral vectors ${ }^{16}$ have been produced in iCELLis Nano. In addition, adenoviral vector production has been scaled-up to iCELLis $500 .^{15}$

Here, for the first time, LV production was optimized for scaleup using iCELLis Nano in perfusion setting in adherent 293T cells. Process was designed to be adaptable to iCELLis 500. Both CaPho precipitation and PEI transfection method were used in transfection, and production conditions, such as perfusion rate, production $\mathrm{pH}$, plasmid concentrations and harvest window were optimized. Although several runs were performed, only 10 most important are reported here. Runs 1-8 describe the main optimization findings, runs 9 and 10 are repeating run 8 , and the remainder of the runs (not described here) were mainly performed to confirm previous observations.

\section{RESULTS}

Cell growth and distribution in iCELLis Nano

The production process in iCELLis Nano bioreactor lasted for 8 days: following inoculation on day 0 , cells were expanded on days 1-3, and transfected on day 4 . Virus collection started 1-day post-transfection (PT; Figure 1) and lasted 2 days. Cultivation

\footnotetext{
${ }^{1}$ A.I. Virtanen Institute for Molecular Sciences, University of Eastern Finland, Kuopio, Finland; ${ }^{2}$ FinVector Vision Therapies, Kuopio, Finland; ${ }^{3}$ Kuopio Center for Gene and Cell

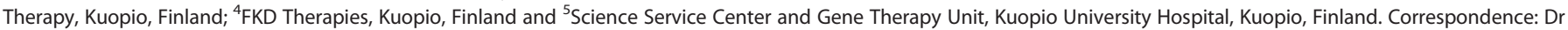
HP Lesch, Research and Development FinVector Vision Therapies, Microkatu 1, Kuopio FI-70210, Finland.

E-mail: hanna.lesch@finvector.com

${ }^{6}$ These authors contributed equally to this work.

Received 2 May 2017; revised 25 September 2017; accepted 29 September 2017; accepted article preview online 5 October 2017 ; advance online publication, 18 January 2018
} 
parameters were continuously recorded and controlled (Supplementary Figure 1). Bioreactor media volume was $700 \mathrm{ml}$ (Table 1). To provide continuous supply of fresh medium, and remove metabolites from the cell culture, as well as to collect the product efficiently, a perfusion system was used. In runs 1-6, fixed perfusion rates were used, whereas in runs 7-10 perfusion rates were adjusted between 0.35 and $4.5 \times$ working volume (wv) per day according to glucose and lactate concentrations (Table 1).

Inoculation cell density target in the bioreactor was 150000 200000 cells $\mathrm{cm}^{-2}$ on day 4 when cells were transfected, as this cell density would be comparable to $70 \%$ confluency in flask experiments. ${ }^{24}$ Cell density was analyzed daily after inoculation

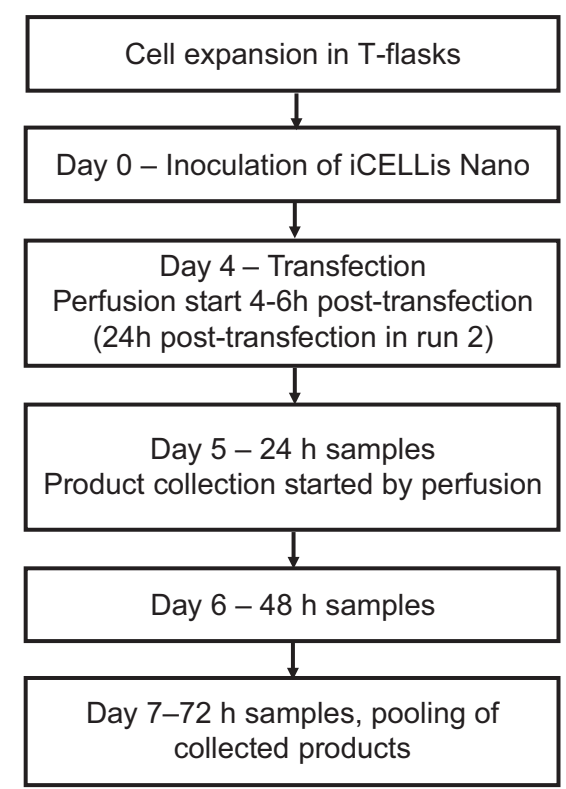

Figure 1. A process flow chart for LV production in iCELLis Nano. from top carriers of the bioreactor bed by counting lysed nuclei. ${ }^{15}$ In previous experiments (data not shown), targeted cell density was exceeded with inoculation amounts between 8000 and 10000 cells $\mathrm{cm}^{-2}$. Thus, in these runs less cells (7000 cells $\mathrm{cm}^{-2}$ ) were inoculated. Despite constant inoculation cell density, top carrier cell counts on day 4 varied between runs (Figure 2a). Except runs $4-5$ and standardized runs 9 and 10, where cell
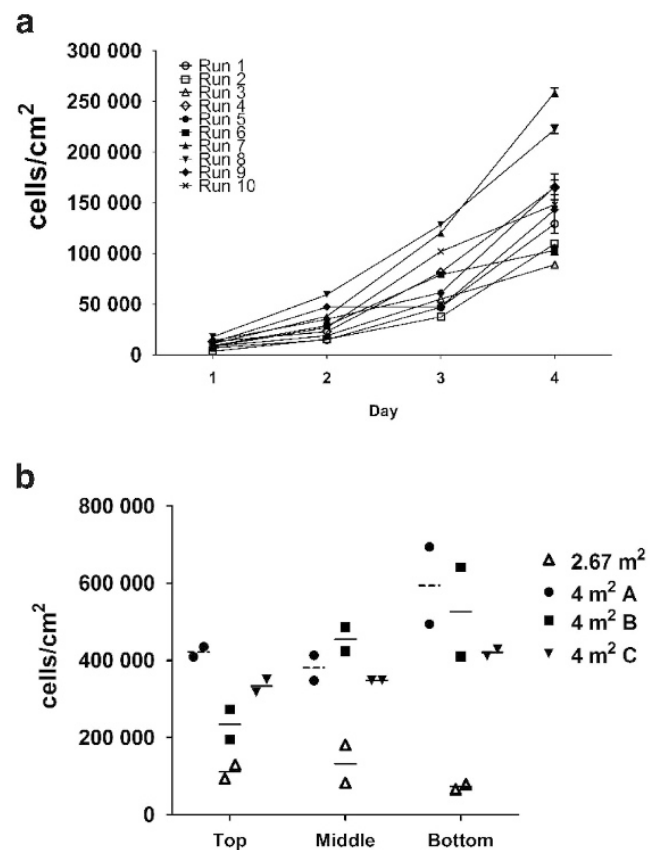

Figure 2. Cell growth and distribution across the bioreactor. (a) Cell growth before transfection as measured from the top carriers, $n=3$ mean \pm s.d. (b) Cell densities counted from top, middle and bottom of four bioreactors; one with $2.67 \mathrm{~m}^{2}$ and three with $4 \mathrm{~m}^{2}$ (a-c) fixedbed sizes, $n=3$ mean \pm s.d.

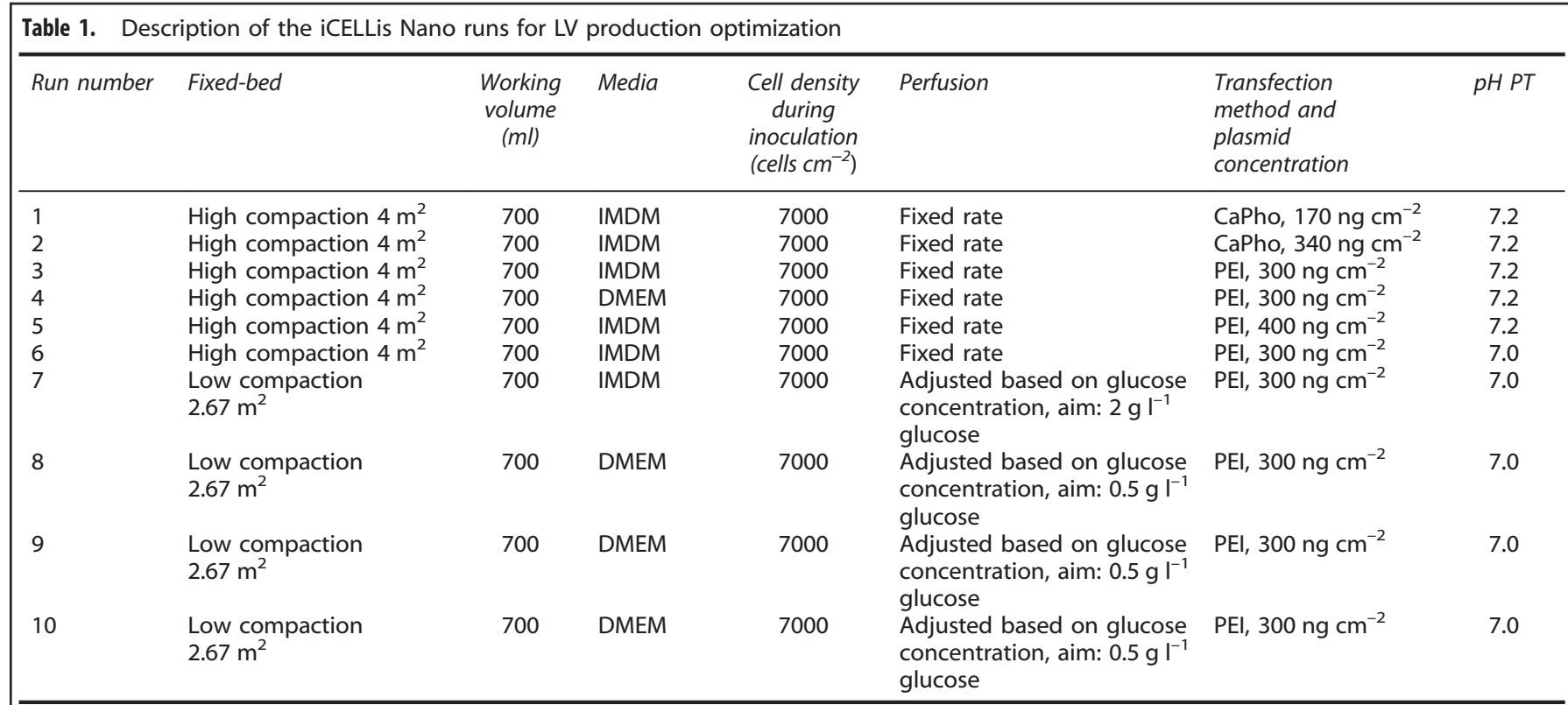

Abbreviations: CaPho, calcium phosphate; DMEM, Dulbecco's modified Eagle's medium; El, polyethylenimine; LV, lentiviral vector; IMDM, Iscove's modified Dulbecco's medium; PT, post-transfection. 
density was on target, transfection cell densities were generally below 150000 cells $\mathrm{cm}^{-2}$ (Figure 2a). In all runs, cells were still actively proliferating $24 \mathrm{~h}$ after transfection, but later cell density increased only slightly (data not shown).

To examine the cell distribution in various parts of $4 \mathrm{~m}^{2}$ fixedbed high compaction bioreactor, several bioreactors were disassembled $72 \mathrm{~h} \mathrm{PT}$, and cell density was analyzed from top, middle and bottom part of the bed (Figure 2b). Stirring rates were identically increased during inoculations to better distribute cells across the bioreactor bed. Variability was seen in cell densities between runs and layers. In $4 \mathrm{~m}^{2}$ bioreactors, cell density was lowest in top carriers and highest in the bottom. Therefore, although the targeted cell density in the top carriers during transfection was not reached in most runs, cell density in the middle or bottom may have been on target. When cells were counted from low compaction $2.67 \mathrm{~m}^{2}$ bioreactor fixed-bed 2 days after inoculation cells seemed to be more evenly distributed (Figure 2b).
Concurrently with iCELLis Nano runs, as 2D controls, triple flasks $\left(500 \mathrm{~cm}^{2}\right.$ ) were seeded using the same cell density (cells $\mathrm{cm}^{-2}$ ). $\mathrm{pH}$ was monitored but not controlled, and perfusion was simulated with timed full medium exchanges. In most control flasks, cell density at transfection clearly exceeded target (over 300000 cells $\mathrm{cm}^{-2}$, data not shown). Cells are more evenly distributed and may be proliferating more rapidly in flasks than on the iCELLis carriers.

Glucose consumption, lactate concentrations and medium usage There was a mild decrease in glucose concentration (Figure 3a) and an increase in lactate concentration (Figure $3 \mathrm{~b}$ ) in all bioreactors before transfection. This change was more pronounced immediately after transfection when virus production started. The highest lactate concentration was seen in run 6 , being $5.86 \mathrm{~g} \mathrm{I}^{-1}$ in the end of the run (Figure 3b). In most runs, lactate
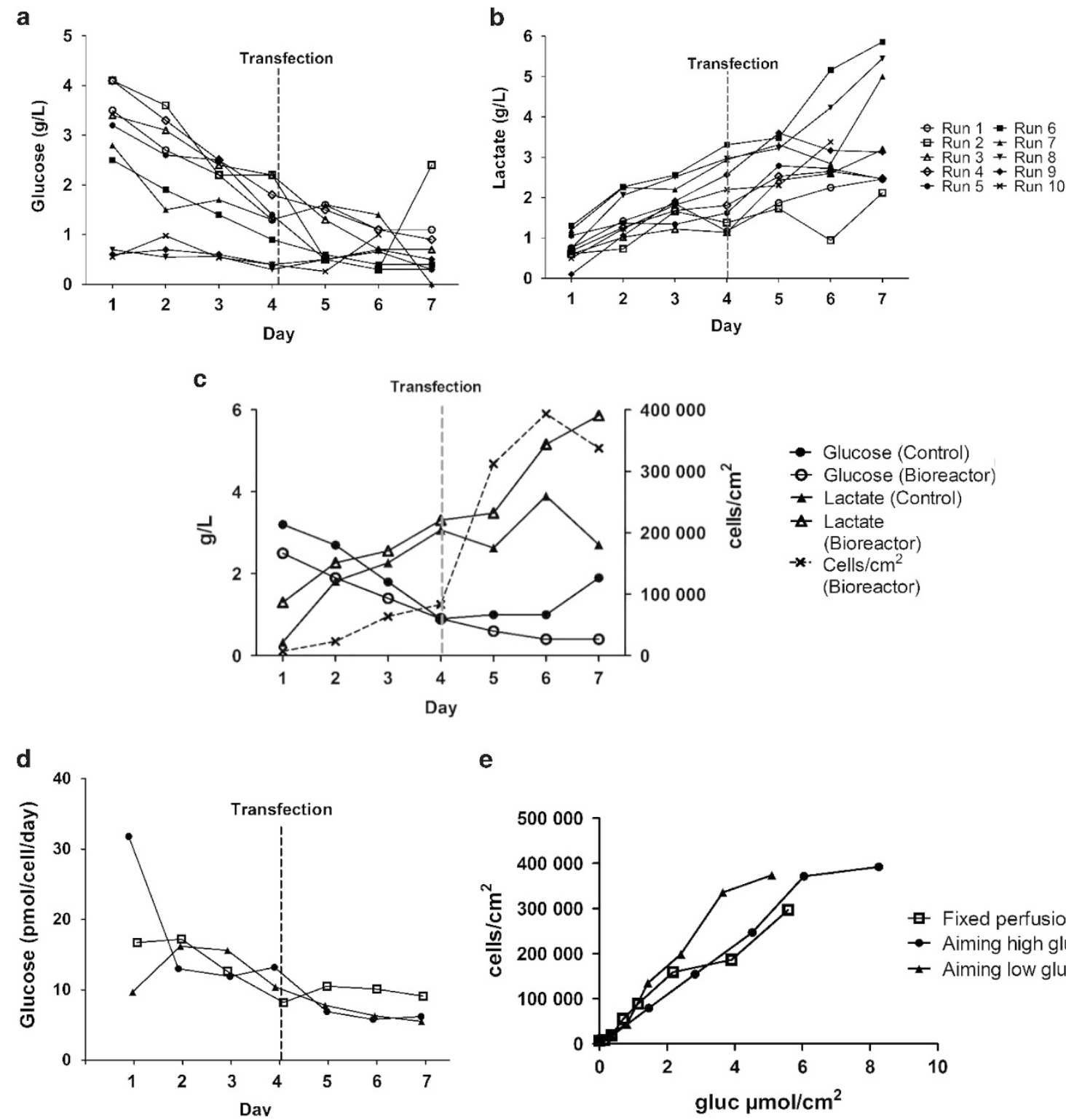

e

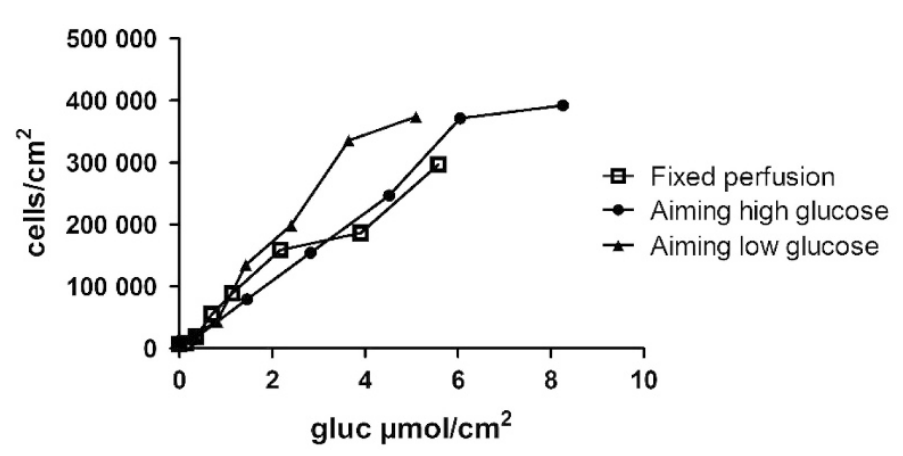

Figure 3. Glucose consumption in iCELLis Nano. (a) Glucose and (b) lactate concentrations measured daily from cell culture medium. (c) Glucose and lactate levels in bioreactor (run 6) and respective control flasks, and cell growth ( $n=3$ mean \pm s.d.) in bioreactor from inoculation to harvest. (d) Glucose consumption per cell per day in run with fixed perfusion rate and in runs when targeting low or high glucose concentration by adjusting perfusion rates. (e) Correlation between cell density and glucose consumption $\mathrm{cm}^{-2}$. 
concentration stayed below $3.5 \mathrm{~g} \mathrm{I}^{-1}$. In addition, lactate concentration seemed to correlate with cell density (Figure 3c).

Similar to the bioreactor runs, glucose concentration started to decrease right after seeding also in the control flasks (Figure 3c). In flasks, only a slight decrease or sometimes even an increase was seen in glucose concentration after transfection, likely due to daily medium changes. There was also some increase in lactate concentrations in these transfected cultures (Figure 3c).

Interestingly, when a run with fixed perfusion rate was compared with runs with adjusted perfusion rates aiming for high $\left(2 \mathrm{~g} \mathrm{I}^{-1}\right)$ or low glucose $\left(0.5 \mathrm{~g} \mathrm{I}^{-1}\right)$, differences in glucose consumption per cell per day were mostly seen during the first $48 \mathrm{~h}$ after inoculation (Figure 3d). Twenty-four hours after inoculation, glucose consumption per cell per day seemed to be highest when high glucose concentration was targeted and lowest when low glucose was targeted. However, at $48 \mathrm{~h}$, after a decline beginning at $24 \mathrm{~h}$, glucose consumption per cell per day was about the same perfusion modes. When fixed perfusion rates were used, it seemed that glucose consumption was somewhat higher compared with when adjusted rates were used. It was also found that when cells were maintained in low glucose medium they required less glucose for their proliferation, compared with cells maintained in high glucose medium (Figure 3e).

Both Iscove's modified Dulbecco's medium (IMDM runs 1-3, 57) and high glucose Dulbecco's modified Eagle's medium (DMEM, runs 4 and $8-10$ ) were used in perfusion during the production (Table 1). Total medium usage for fixed perfusion rate runs (runs 1-6) was approximately 10 liters (4 liters before and 6 liters PT). In runs $1-5$, medium in the bioreactors was completely changed on days 5 and 6 resulting in additional consumption of 1.5 liters medium PT. Therefore, the product volume in runs 1-5 was approximately 7.5 liters and in run $6, \sim 5.5$ liters. When aiming for high glucose in low compaction bioreactor a total of $\sim 15$ liters medium was used for the perfusion, with $\sim 9$ liters for product. Nevertheless, in that run glucose concentration was lower than targeted and lactate levels increased considerably. Therefore, the actual medium usage required to maintain such a high glucose concentration would likely be much higher. Perfusion media consumption was reduced by targeting low glucose in low compaction bioreactor (runs 8-10) to 5-7 liters with a product volume of $3-5$ liters, without affecting productivity.

\section{CaPho transfection}

293T cells were transiently transfected with four plasmids required for third-generation LV production. CaPho precipitation method, traditionally used for transfections in 2D systems, was tested in bioreactors first. First, CaPho transfection was used identically to small-scale production methods with the exception that only half of the plasmid amount $\mathrm{cm}^{-2}$, as compared with production of LV in flasks, was used to ensure scalability of the process to the bioreactor. ${ }^{13}$ Several runs were performed, however, run 1 was chosen to represent the results of the preliminary scale-up process. Later, the performance was enhanced by increasing plasmid concentrations equivalent to those used for LV production in flasks. In run 2, the transfection mixture was recirculated to find the optimal conditions for large-scale transfection with CaPho method. Recirculation loop was replaced with perfusion $24 \mathrm{~h}$ PT.

Fluorescence microscopy (Supplementary Figure 2) and flow cytometry were used to analyze the transfection efficiencies by measuring the distribution and percentage of green fluorescent protein (GFP)-positive cells $24 \mathrm{~h}$ PT. Transfection efficiencies varied a lot between runs (data not shown) both because of differences in transfection protocol and difficulties in detaching the cells from carriers.

In order to follow the productivity, bioreactors and control flasks were sampled at 24, 48 and $72 \mathrm{~h}$ PT (Figure 4) for analysis of total functional particles (TU) and total viral particles (vp). Total LV yield was analyzed from the medium collected for approximately 2 days starting 24-h PT (Table 2).

The highest functional titers were obtained at 48-h time point both in flasks and in the bioreactor. However, the difference to 24$\mathrm{h}$ and 72-h time points was minor, with relatively high titers already at 24-h PT (Figure 4). The CaPho transfected culture was a

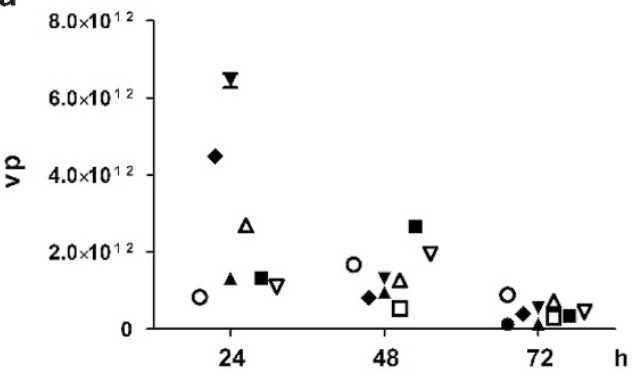

C

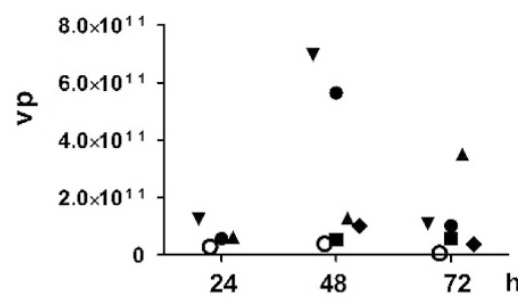

b

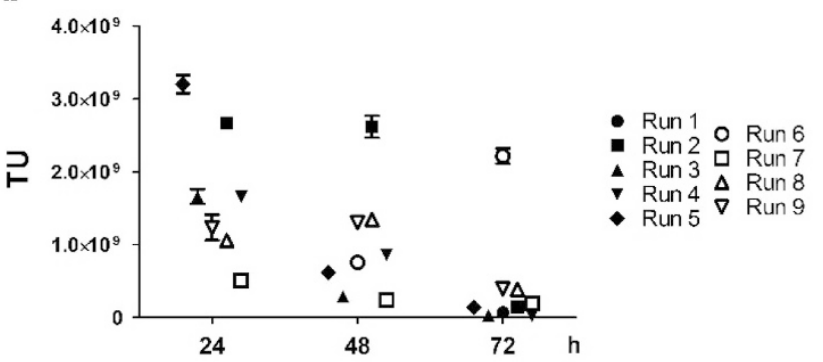

d

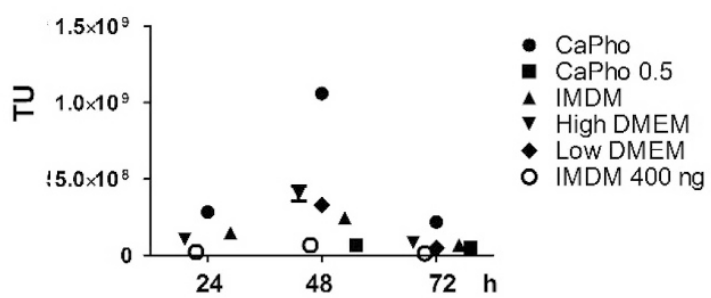

Figure 4. Total yields in the bioreactor and control flasks in $24-, 48-$ and 72-h time points PT. (a, b) Total vp and TU, respectively, in bioreactors, mean \pm s.d. Bioreactor of run 10 was not sampled. (c, d) Total vp and TU, respectively, in controls, mean \pm s.d. CaPho precipitation with regular plasmid amount $\left(340 \mathrm{ng} \mathrm{cm}^{-2}\right.$ ), IMDM as medium; CaPho $1 / 2=\mathrm{CaPho}$ transfection with smaller plasmid amount $\left(170 \mathrm{ng} \mathrm{cm}^{-2}\right.$ ), IMDM as medium; IMDM = PEI transfection, IMDM medium; high DMEM=PEI transfection, high glucose DMEM as medium; low DMEM=PEI transfection, low glucose DMEM as a medium; IMDM $400 \mathrm{ng}=$ PEl transfection with higher plasmid amount (400 ng); IMDM as medium. PT, post-transfection; TU, transducing units; vp, viral particles. 
also sampled once at 96-h PT, however, at that time titers had decreased significantly (results not shown).

The best yields of CaPho runs (total vp 7.18E+12 and total TU 8.57E+09) were obtained in run 2 with optimized transfection and higher plasmid concentration. However, despite the doubled plasmid amounts compared with run 1, total TU and productivity in $\mathrm{TU} \mathrm{cm} \mathrm{cm}^{-2}$ were only 1.7 times higher, whereas in control flasks, when the plasmid amounts were doubled in CaPho-mediated transfection, $\mathrm{TU} \mathrm{cm} \mathrm{cm}^{-2}$ increased 10 -fold, and $\mathrm{vp} \mathrm{cm}^{-2}$ was also increased (Table 3). Thus, large-scale production cannot be directly scaled-up by simply increasing plasmid concentration. In addition, owing to known difficulties in performing transfection as reviewed in McCarron et al. ${ }^{25}$ and inter-run productivity variation, optimization was continued with PEl-mediated.

\section{PEI transfection}

Before using PEI in iCELLis Nano, PEI transfection was optimized using $175 \mathrm{~cm}^{2}$ flasks. Transfection efficiency measured 1-day PT was almost $100 \%$ when PEl was used in transfections with 300 and $400 \mathrm{ng} \mathrm{cm}^{-2}$ plasmid DNA. With $100 \mathrm{ng} \mathrm{cm}^{-2}$, efficiency dropped to $5 \%$. Total vp produced in flasks transfected with $300 \mathrm{ng} \mathrm{cm}^{-2}$ was about $1.5 \times$ higher compared with $400 \mathrm{ng} \mathrm{cm}^{-2}$. Transfection with $1: 1$ and $1: 1.5(\mu \mathrm{g}$ DNA):( $\mu \mathrm{PEI}$ ) ratio yielded the best efficiency with more vps produced with 1:1 ratio. Transfection was unsuccessful with 1:0.5 ratio and with 1:2 ratio transfection efficiency began to slightly decrease. Thus, $300 \mathrm{ng} \mathrm{cm}^{-2}$ and DNA: PEI ratio of 1:1 were used in the first iCELLis Nano runs with PEI transfection.

PEI transfection was easier to perform and more robust in the bioreactor because of less stringent condition requirements compared with CaPho precipitation based on repeated runs 8-10. In bioreactors, PEI transfection efficiencies (\% of GFPpositive cells) measured from top carriers $24-\mathrm{h}$ PT varied between 43 and $80 \%$, and when transfection efficiency of $4 \mathrm{~m}^{2}$ fixed-bed

\begin{tabular}{|c|c|c|c|c|c|}
\hline Run number & Total vp & $v p \mathrm{~cm}^{-2}$ & Total TU & $T U \mathrm{~cm}^{-2}$ & $T U n g^{-1} p 24$ \\
\hline 1 & $5.24 \mathrm{E}+12$ & $1.31 \mathrm{E}+08$ & $5.09 E+09$ & $1.27 \mathrm{E}+05$ & 12127 \\
\hline 2 & $7.18 \mathrm{E}+12$ & $1.79 \mathrm{E}+08$ & $8.57 \mathrm{E}+09$ & $2.14 \mathrm{E}+05$ & 14924 \\
\hline 3 & $7.38 \mathrm{E}+12$ & $1.84 \mathrm{E}+08$ & $2.83 \mathrm{E}+09$ & $7.08 \mathrm{E}+04$ & 4798 \\
\hline 4 & $9.73 \mathrm{E}+12$ & $2.43 \mathrm{E}+08$ & $2.09 \mathrm{E}+09$ & $5.23 \mathrm{E}+04$ & 2771 \\
\hline 5 & $1.75 \mathrm{E}+13$ & $4.38 \mathrm{E}+08$ & $5.20 E+09$ & $1.30 \mathrm{E}+05$ & 3715 \\
\hline 6 & $1.48 \mathrm{E}+13$ & $3.71 \mathrm{E}+08$ & $1.15 \mathrm{E}+10$ & $2.89 \mathrm{E}+05$ & 10017 \\
\hline 7 & $1.30 \mathrm{E}+13$ & $4.85 \mathrm{E}+08$ & $9.70 E+09$ & $3.63 E+05$ & 9361 \\
\hline 8 & $9.01 \mathrm{E}+12$ & $3.37 E+08$ & $9.85 \mathrm{E}+09$ & $3.69 \mathrm{E}+05$ & 13669 \\
\hline 9 & $7.42 \mathrm{E}+12$ & $2.78 \mathrm{E}+08$ & $5.79 \mathrm{E}+09$ & $2.17 \mathrm{E}+05$ & 9757 \\
\hline 10 & $8.44 \mathrm{E}+12$ & $3.16 \mathrm{E}+08$ & $7.86 \mathrm{E}+09$ & $2.94 \mathrm{E}+05$ & 12520 \\
\hline
\end{tabular}

(without virus production) was measured at 72-h PT almost 100\% of cells were transfected. When transfection efficiency was measured from different layers at 72-h time point, it was found that transfection efficiency is slightly higher in top carriers $(99.9 \%$ vs $99.5 \%$ ) compared with bottom and middle carriers. Also strength of the GFP expression was higher in top carriers. Productivities between runs did not vary as much as between CaPho runs.

The first two PEI runs (runs 3 and 4) were performed using $300 \mathrm{ng} \mathrm{cm}^{-2}$ of plasmid DNA. IMDM and DMEM were used for perfusion in runs 3 and 4, respectively. Aim was to maintain relatively high glucose and low lactate concentration, with fixed perfusion rates. Total TU produced was rather low in these runs, and based on $\mathrm{TU} \mathrm{ng}^{-1}$ p24 ratio it seemed that more nonfunctional vp relative to functional particles were produced (Table 2).

Increased plasmid concentration $\left(400 \mathrm{ng} \mathrm{cm}^{-2}\right)$ in PEI transfection was tested during run 5, with IMDM perfusion. Higher plasmid concentration seemed to increase the total vp (total $1.75 \mathrm{E}+13 \mathrm{vp}$; Table 2). This effect was not seen in flasks. However, in the bioreactor the total TU obtained was only $5.20 \mathrm{E}+09$ and thus $\mathrm{TU} \mathrm{ng}^{-1}$ p24 ratio was low.

In most PEl runs, the highest number of $\mathrm{vp}$ in the bioreactor were seen already $24-h$ PT, being almost 0.5 or $1-2$ log lower $48-h$ PT and 72-h PT, respectively (Figure 4a). Similarly, the highest functional titer in PEI runs was obtained already within the first $24 \mathrm{~h}$ (Figure 4b). On the other hand, samples taken 6-h PT did not show remarkable productivities (results not shown) and therefore the harvest window was determined to fall between $24-h$ and 72-h PT.

Lowering $\mathrm{pH}$ after transfection increases LV yields

In runs $3-5, \mathrm{pH}$ was maintained at 7.2. Lower $\mathrm{pH}$ has been shown to increase LV production. ${ }^{26}$ Therefore, in runs $6-10 \mathrm{pH}$ was decreased to 7.0 after PEl-mediated transfection. Lower $\mathrm{pH}$ had a positive impact on functional titer, with the total TU exceeding $1 \mathrm{E}$ +10 for the first time (Table 2). In addition to improved TU $\mathrm{ng}^{-1}$ p24 ratio, total vp was slightly higher than in comparable runs 3-4 but lower than in the previous run (run 5) with higher plasmid concentration.

Using low compaction bioreactor and adjusting perfusion rate based on glucose concentration decreased total medium consumption but maintained productivity

Glucose consumption and lactate concentration were high in run 6 , especially after transfection. Therefore, we decided to adjust the perfusion rates based on glucose and lactate concentrations. With fixed perfusion rates, glucose concentration tended to drop below $1 \mathrm{~g} \mathrm{I}^{-1}$ after transfection. Thus, targeting high glucose $\left(2 \mathrm{gl}^{-1}\right)$ in $4 \mathrm{~m}^{2}$ bioreactor was likely to increase the total medium consumption. Therefore, a low compaction bed $\left(2.67 \mathrm{~m}^{2}\right)$ was

Table 3. Yields obtained in $2 \mathrm{D}$ control flasks

\begin{tabular}{|c|c|c|c|c|c|c|}
\hline IMDM & CaPho $170 \mathrm{ng} \mathrm{cm}^{-2}$ & $1.11 \mathrm{E}+11$ & $2.21 \mathrm{E}+08$ & $1.18 \mathrm{E}+08$ & $2.35 E+05$ & 13313 \\
\hline IMDM & CaPho $340 \mathrm{ng} \mathrm{cm}^{-2}$ & $6.66 \mathrm{E}+11$ & $1.33 \mathrm{E}+09$ & $1.28 \mathrm{E}+09$ & $2.55 \mathrm{E}+06$ & 23946 \\
\hline IMDM & PEI $400 \mathrm{ng} \mathrm{cm}^{-2}$ & $4.55 E+10$ & $8.66 \mathrm{E}+07$ & $7.79 \mathrm{E}+07$ & $1.48 \mathrm{E}+05$ & 21414 \\
\hline High glucose DMEM & PEI $300 \mathrm{ng} \mathrm{cm}^{-2}$ & $8.05 E+11$ & $1.53 \mathrm{E}+09$ & $1.93 \mathrm{E}+08$ & $3.68 \mathrm{E}+05$ & 2992 \\
\hline Low glucose DMEM & PEI $300 \mathrm{ng} \mathrm{cm}^{-2}$ & $1.38 \mathrm{E}+11$ & $2.62 \mathrm{E}+08$ & $3.74 \mathrm{E}+08$ & $7.12 \mathrm{E}+05$ & 33924 \\
\hline
\end{tabular}


used in run 7. Interestingly, despite the smaller bed size, total TU produced was almost as good as in the previous run with high compaction bed. Indeed, $3.63 \mathrm{E}+05 \mathrm{TU} \mathrm{cm}^{-2}$ and $4.85 \mathrm{E}+08 \mathrm{vp} \mathrm{cm}{ }^{-2}$, the highest titers so far were achieved in run 7 (Table 2).

High glucose concentration target increases medium consumption and total product volume. Thus, we next applied low glucose target $\left(0.5 \mathrm{gl}^{-1}\right)$ for the low compaction bioreactor. Cells were inoculated in low glucose DMEM and grown with low perfusion rates using high glucose DMEM. As a result, perfusion rate, glucose concentration and consequently cell growth were easier to control. The product volume was reduced, but importantly yields as well as the $\mathrm{TU} \mathrm{ng}^{-1}$ p24 ratio remained high. In control flasks, similarly to the bioreactors, the lowest $\mathrm{TU} \mathrm{ng}^{-1}$ p24 ratio was obtained when cells were grown in high glucose DMEM, whereas the best yield and $\mathrm{TU} \mathrm{ng}^{-1}$ p24 ratio were obtained when cells were grown in low glucose DMEM (Table 3). However, in the bioreactors productivity $\mathrm{cm}^{-2}$ was one log lower compared with flasks (Tables 2 and 3 ).

\section{DISCUSSION}

According to the Gene Therapy Clinical Trials Worldwide database provided by the Journal of Gene Medicine, there is a growing interest in LVs, and they are considered as a promising vector type for gene therapy. ${ }^{1,2}$ However, LV production methods need to be upgraded for efficient large-scale production for clinical applications. Here, we optimized production methods for scalable LV production in a fixed-bed bioreactor. Two different scalable transfection methods widely used in LV production ${ }^{25}$ were tested, that is, CaPho precipitation and PEl-mediated transfection, of which PEl appeared more convenient in large-scale production in terms of scalability and reproducibility. In addition, PElpro used in this study is available as good manufacturing practices compliant material. We continued optimizing PEI transfection and the overall LV production process in the bioreactor by determining the best production conditions and equipment for our process leading to scalable high-titer LV production.

The fixed-bed of iCELLis bioreactors consists of polyester microfiber macrocarriers. At the time low and high compaction, fixed-bed bioreactors were available. High cell densities have been shown to correlate with high LV productivities, ${ }^{17}$ and high compaction fixed-bed with a larger growth area was expected to produce higher titers compared with low compaction. However, in our experiments cell densities in low compaction fixed-bed were higher compared with most of the runs in high compaction, the productivity $\mathrm{cm}^{-2}$ and total yields were highest among the low compaction runs. Cell count on samples from different vertical and horizontal layers of bed suggested that high compaction fixedbed was probably too dense for an even cell distribution, and likely suffered from channeling of the fixed-bed packaging material resulting in uneven distribution of the cells. Cell distribution was more even in low compaction fixed-bed, which suggested that the fixed-bed material was more evenly distributed and channeling of the low compaction fixed-bed was minor. Beds were not disassembled at the same phases of the production, which may have affected the results. However, transfection efficiencies and productivities $\mathrm{cm}^{-2}$, as well as total costs of the process confirmed that the low compaction is more favorable for efficient LV production. Similar findings have been reported previously. ${ }^{15,16,23}$

In addition to daily cell count from top carriers, cell growth was monitored by measuring glucose and lactate concentrations from the bioreactor. Glucose consumption and lactate accumulation in the bioreactor increased during the first runs performed with constant perfusion rates. This indicated that constant perfusion and daily full medium exchange were not sufficient to control the glucose levels in the bioreactor. Low glucose concentrations, however, had no major effect on LV production. In addition, cells seemed to adapt well to the low glucose concentration, and exhibited cell growth comparable with growth in high glucose medium. Therefore, aiming at low glucose in large-scale runs by changing perfusion rates accordingly, and using high glucose medium for perfusion would decrease the total medium and thus also fetal bovine serum (which availability is diminishing) consumption, product volume and therefore also production costs. Reducing the product volume increases control over the product and alleviates downstream purification. In later runs with controlled perfusion rate to maintain the targeted glucose concentration, lactate concentrations increased to relatively high levels. This could be disadvantageous because of decreasing $\mathrm{pH}$, however, with constant $\mathrm{pH}$ control increasing lactate concentrations did not affect the LV production.

Lower $\mathrm{pH}$ levels close to $\mathrm{pH} 6$ have been shown to increase vesicular stomatitis virus envelope glycoprotein $\mathrm{G}$ pseudotyped LV productivity. ${ }^{26}$ As both cells and LV particles are sensitive to $\mathrm{pH}$ changes, the correct $\mathrm{pH}$ for LV production is determined by finding the optimal $\mathrm{pH}$ value for efficient LV production in the cells in question. In control flasks, pH decreased below 7.0 by the end of culture because of lack of $\mathrm{pH}$ control, which indicates that the $\mathrm{pH}$ could be decreased also in the bioreactor. Indeed, we obtained the highest LV productivities ( $\mathrm{TU} \mathrm{cm}^{-2}$ ) and total TU amounts when $\mathrm{pH}$ set point was decreased to 7.0. Lower $\mathrm{pH}$ set point also improved the ratio of functional particles to p24 protein.

For transfection, we first tested CaPho precipitation method traditionally used in 2D systems. Although CaPho precipitation has been successfully used to transfect 293T cells for producing LV in small scale, there were difficulties in using CaPho in iCELLis Nano. In control flasks, CaPho led to higher functional titers than PEl, but bioreactor runs were not easily reproducible, and transfection method itself was difficult and impractical to execute. This is likely due to the sensitivity of the CaPho method to adherence to protocols established for smaller volumes and its intolerance to variations caused by preparation and transfer of larger transfection volumes. Production using PEI transfection has been reported to be similar or lower than with CaPho precipitation, ${ }^{18}$ however, the method itself was reported to be easier to perform, ${ }^{27}$ as it involves less critical variables and less reagents. Therefore, in later runs, we decided to use $\mathrm{PEI}$ in transfections.

In our experiments, optimal DNA:PEI ratio for the production was the only critical parameter for productivity. We were able to achieve the productivity of CaPho runs with PEI transfection by using less DNA than was required for CaPho precipitation. In fact, smaller DNA concentration has been reported to generally result in higher titers, probably because of reduced cytotoxicity. ${ }^{17,28} \mathrm{We}$ also discovered that higher plasmid concentration does not result in an equivalent increase in productivity. A moderate increase in titers was observed but this does not compensate for the costs of the additional plasmids. Overall, the reproducibility was better, and the differences in productions between runs performed with different parameters were smaller between PEI runs than CaPho runs. These findings confirm the general opinion that PEI transfection is preferable when considering large-scale manufacturing of LV. $9,17,18$

Correctly timed harvest is important in well-designed LV production. A poorly designed harvest window results either in diluted product and increasing downstream expenses, or decreased product output if the window is too narrow. LV collection begins generally ${ }^{13} 2$-day PT to ensure complete removal of the transfection reagents. Here, harvest window was between 24 and 72-h PT and titers were high already in the beginning of harvest. However, the titers were still very low at the time of PT perfusion initiation. Thus, the optimal harvest could be started after PT perfusion initiation, but earlier than 24-h PT.

Productivity $\mathrm{cm}^{-2}$ was higher in the flasks compared with bioreactors. This might result from the faster proliferation of the cells in the flasks resulting in higher cell concentrations. On the 
other hand, owing to higher inoculation concentrations (cells $\mathrm{ml}^{-1}$ ) in the bioreactor, cells in lower parts of the fixed-bed may end up attaching to each other into large clusters, which may be inaccessible to transfection reagents. This was not observed on the top carriers but cell densities are higher in the lower parts of the fixed-bed. Although the productivity $\mathrm{cm}^{-2}$ was higher in control flasks, in terms of total TUs, average bioreactor run in iCELLis Nano was equal to production in approximately 30 triple flasks, and at best equal to over 100 triple flasks. Approximation was obtained from optimization runs with varying conditions, and higher yields can be assumed in standardized production. Replacing flask production with a highly integrated bioreactor system reduces labor demands and costs notably.

Scaled-up to iCELLis 500 with low compaction fixed-bed of $333 \mathrm{~m}^{2}, 1$-week production in $2.67 \mathrm{~m}^{2}$ iCELLis Nano (about 6 liters medium consumption for perfusion, $\sim 2.16 \mathrm{E}+06 \mathrm{TU} \mathrm{ml}^{-1}$ in 4 liters, $7.86 \mathrm{E}+09$ total TU) would correspond to 750 liters medium for perfusion instead of almost 2000 liters when targeting high glucose, $\sim<00$ liters product volume and $1.1 \times 10^{12} \mathrm{TU}$ in total. Cell expansion for inoculating the $333 \mathrm{~m}^{2}$ bioreactor could be carried out in large-scale 2D cell culture vessels, available, for example, in sizes of $1720 \mathrm{~cm}^{2}$. Cell expansion for 4 days in the bioreactor allows decreased inoculation amounts, thus decreasing the amount of material and working hours needed to expand the cells before inoculation.

Titers and total TU obtained in this study are comparable with current LV pseudotyped with the vesicular stomatitis virus envelope glycoprotein $\mathrm{G}$ adherent production methods. However, titers in terms of $\mathrm{TU} \mathrm{ml}^{-1}$ are not directly comparable with other studies as various factors affect the results, including different plasmid constructs and titering protocols. ${ }^{29}$

In conclusion, we were able to improve current working methods by creating a scalable process for efficient LV production. The bioreactor used was compact and facilitated the production comparable to $2 \mathrm{D}$ production. Utilizing the working methods presented here, LV production can be scaled-up to provide sufficient amounts of material for clinical applications.

\section{MATERIALS AND METHODS}

Cell culture

$293 \mathrm{~T}$ cells (ATCC, Manassas, VA, USA) were cultivated at $37^{\circ} \mathrm{C}$ and $5 \% \mathrm{CO}_{2}$ in high or low glucose DMEM (Sigma-Aldrich, Irvine, UK) supplemented with $10 \%(\mathrm{v} / \mathrm{v})$ fetal bovine serum (Sigma-Aldrich, St Louis, MO, USA/Gibco, Paisley, UK) and $50-100 \mathrm{U} \mathrm{ml}^{-1}$ penicillin, $50-100 \mathrm{\mu g} \mathrm{ml}^{-1}$ streptomycin (Sigma-Aldrich) and $2 \mathrm{mM}$ L-glutamine (Gibco/Sigma-Aldrich). Similarly, supplemented IMDM (Sigma-Aldrich) was used in LV production (see Tables 1 and 3).

\section{Production conditions in bioreactor}

LV production was performed in iCELLis Nano fixed-bed bioreactor (Pall Corporation, Brussels, Belgium). Used fixed-bed areas were $2.67 \mathrm{~m}^{2}$ (bed height $10 \mathrm{~cm}$, carrier compaction $96 \mathrm{~g} \mathrm{I}^{-1}$ ) and $4 \mathrm{~m}^{2}$ (bed height $10 \mathrm{~cm}$, carrier compaction $144 \mathrm{~g} \mathrm{I}^{-1}$ ). iCELLis is equipped with a magnetic stirrer for mixing and lifting medium, cells and transfection reagents up the carrier bed. Gas exchange takes place in the headspace, and oxygenated medium is recirculated back to cells. Working volume of the bioreactor was adjusted to $700-800 \mathrm{ml}$. Bioreactor control unit communicated with my-Control software (Applikon Biotechnology, Schiedam, Netherlands), and the cultivation data was collected with BioXpert V2 (Applikon Biotechnology).

Bioreactor was equipped with $\mathrm{pH}$ control set to 7.2 during runs $1-5$. Set point was decreased to $7.0 \mathrm{PT}$ in runs $6-10$. The $\mathrm{pH}$ value was maintained with $\mathrm{CO}_{2}$ and $7.5 \%$ sodium bicarbonate (Sigma-Aldrich), and online value was controlled daily with offline measurements, with a $3 \mathrm{ml}$ fresh sample from the bioreactor. Stirring was set to $1-2.65 \mathrm{~cm} \mathrm{~s}^{-1}$ medium linear speed, with higher stirring during inoculation and transfection. Dissolved oxygen was maintained at $50 \%$ with air and oxygen supply, and temperature at $37^{\circ} \mathrm{C}$. Cell growth was monitored daily by sampling top carriers $(n=3)$, which were lyzed into a nucleus suspension, and counting nuclei similarly to Lesch et al. ${ }^{15}$ Cells were also counted from the top, middle and bottom carriers from two sites on different sides of the column from both 4 and $2.67 \mathrm{~m}^{2}$ bioreactors. Glucose and lactate concentrations were measured either once (when using fixed perfusion rates) or twice a day (when adjusting perfusion based on glucose concentration) with a reflectometer (RQflex 10, Merck Millipore, Darmstadt, Germany).

Cells were inoculated at 7000 cells $\mathrm{cm}^{-2}$ on day 0 and transfected on day 4. The harvest was initiated on day 5 and continued until day 7 (Figure 1). Perfusion was performed with high glucose DMEM or IMDM prepared similarly to the cell growth media. At first, perfusion was adjusted to change medium at fixed rates of $1.1-2.9 \times w^{2}$ day $^{-1}$ with a higher PT rate. During the later runs, perfusion rate was adjusted between 0.5 and $4.5 \times$ wv day $^{-1}$ according to the glucose and lactate concentrations, which were measured twice a day, to reach the glucose target value of 2 or $0.5 \mathrm{~g} \mathrm{I}^{-1}$. PT perfusion was initiated on day 4, or day 5 in case of recirculation during transfection. At 24-h PT, perfusion was continued without fetal bovine serum until the end of production. In runs 1-5, medium was completely exchanged in the bioreactor on days 5 and 6.

\section{Transfection}

Third-generation self-inactivating LV expressing GFP under the human PGK promoter ${ }^{30}$ were produced using a four-plasmid system (pVSVg, pGag-Pol, pRev and LV plasmid expressing GFP). Plasmids were manufactured by PlasmidFactory (Bielefeld, Germany) or extracted with EndoFree Plasmid Kit (Qiagen, Hilden, Germany) according to the manufacturer's instructions. Cells were transfected 4 days post-inoculation either with $\mathrm{CaPho}^{13}$ or PElpro (Polyplus, Illkirch, France). A total of $170-400 \mathrm{ng} \mathrm{cm}^{-2}$ plasmid was used. In run 2, bioreactor was equipped with recirculation during transfection in which half of the transfection mixture was added to the bioreactor and the other half to recirculating medium, and the transfection mixture was recirculated through the bioreactor. Runs 9 and 10 were repeat runs of run 8 .

PEI transfection was first optimized in T175 flasks. Four different DNA concentrations, 100, 200, 300 and $400 \mathrm{ng} \mathrm{cm}^{-2}$, and four different DNA:PEI $(\mu \mathrm{g}: \mu \mathrm{l})$ ratios, (1:0.5), (1:1), (1:1.5) and (1:2) were tested. 293T cells were seeded, and transfected on the following day according to the manufacturer's instructions using LV plasmid expressing-GFP ${ }^{31}$ and transfection efficiency was analyzed 24-h PT. The procedure was repeated using four LV production plasmids in two best performing conditions and produced vp was measured. ${ }^{13}$ When performing transfection in bioreactor, DNA and PEI were separately mixed with serum-free culture medium, mixtures were combined and incubated at room temperature. Volume corresponding to transfection mixture was removed from the bioreactor and mixture was added into the bioreactor.

\section{Determination of transfection efficiency and titers}

For determination of transfection efficiency in the bioreactor, the top carriers $(n=5)$ were sampled before transfection and 1-day PT and were viewed under a fluorescence microscope. In addition, trypsin-EDTA (SigmaAldrich, St Louis, MO, USA) was used to detach cells and the percentage of GFP-positive cells was analyzed with flow cytometry (FACSCalibur, Becton Dickinson, Franklin Lakes, NJ, USA).

For determination of the LV titer, $5 \mathrm{ml}$ was sampled from the bioreactor and aliquoted to $1 \mathrm{ml}$ samples. For vp titer, p24 capsid protein was measured with Alliance HIV-1 p24 antigen ELISA kit (PerkinElmer, Waltham, MA, USA) in duplicates. p24 concentrations were transformed to vp titers by assuming $12500 \mathrm{LV}$ particles per $1 \mathrm{pg}$ of p24. ${ }^{31,32}$ Functional particle analysis was performed in duplicates with flow cytometry using HeLa cells. ${ }^{13}$ The mean values of the duplicates with s.d. are reported.

\section{Control flasks}

Concurrently with the bioreactor runs, triple flasks were seeded, sampled $(3 \mathrm{ml})$ for glucose and lactate, and transfected. In order to simulate the perfusion rate in the bioreactor, medium was changed a day after inoculation, before transfection and daily after transfection. $\mathrm{pH}$ was measured daily, but not controlled. For determination of the LV titer, $5 \mathrm{ml}$ was sampled from the flasks and aliquoted to $1 \mathrm{ml}$ samples. Produced $\mathrm{vp}$ and TU were analyzed at different time points after transfection by p24 ELISA and flow cytometry in duplicates, respectively. The mean values of the duplicates with s.d. are reported. 


\section{CONFLICT OF INTEREST}

AJV is an employee of University of Eastern Finland and FinVector Vision Therapies; HML, VT and JM are employees of FinVector Vision Therapies; EML and HPL are employees of Finvector Vision Therapies and FKD Therapies. No conflict of interest exists for TH and SYH.

\section{ACKNOWLEDGEMENTS}

This work was financially supported by Finnish Cultural Foundation North Savo regional Fund. We thank the following people for help and support: lina Laaksonen, Sanna Pietikäinen, Jaana Siponen, Anne Martikainen, Tuula Salonen and Maarit Mähönen. We also thank Nihay Laham Karam for the critical review of the manuscript.

\section{REFERENCES}

1 Ginn SL, Alexander IE, Edelstein ML, Abedi MR, Wixon J. Gene therapy clinical trials worldwide to 2012 - an update. J Gene Med 2013; 15: 65-77.

2 Gene Therapy Clinical Trials Worldwide. Available at: http://www.wiley.com// legacy/wileychi/genmed/clinical// (accessed 19 July 2017).

3 Naldini L, Blömer U, Gallay P, Ory D, Mulligan R, Gage FH et al. In vivo gene delivery and stable transduction of nondividing cells by a lentiviral vector. Science 1996; 272: 263-267.

4 Castiello MC, Scaramuzza S, Pala F, Ferrua F, Uva P, Brigida I et al. B-cell reconstitution after lentiviral vector-mediated gene therapy in patients with Wiskott-Aldrich syndrome. J Allergy Clin Immunol 2015; 136: 692-702.e2.

5 Aiuti A, Biasco L, Scaramuzza S, Ferrua F, Cicalese MP, Baricordi C et al. Lentiviral hematopoietic stem cell gene therapy in patients with Wiskott-Aldrich syndrome. Science 2013; 341: 1233151.

6 Biffi A, Bartolomae CC, Cesana D, Cartier N, Aubourg P, Ranzani M et al. Lentiviral vector common integration sites in preclinical models and a clinical trial reflect a benign integration bias and not oncogenic selection. Blood 2011; 117: 5332-5339.

7 Hacein-Bey-Abina S, Garrigue A, Wang GP, Soulier J, Lim A, Morillon E et al. Insertional oncogenesis in 4 patients after retrovirus-mediated gene therapy of SCID-X1. J Clin Invest 2008; 118: 3132-3142.

8 MacGregor RR. Clinical protocol. A phase 1 open-label clinical trial of the safety and tolerability of single escalating doses of autologous CD4 T cells transduced with VRX496 in HIV-positive subjects. Hum Gene Ther 2001; 12: 2028-2029.

9 Segura MM, Garnier A, Durocher Y, Coelho H, Kamen A. Production of lentiviral vectors by large-scale transient transfection of suspension cultures and affinity chromatography purification. Biotechnol Bioeng 2007; 98: 789-799.

10 Russell WC, Graham FL, Smiley J, Nairn R. Characteristics of a human cell line transformed by DNA from human adenovirus type 5. J Gen Virol 1977; 36: 59-72.

11 DuBridge RB, Tang P, Hsia HC, Leong PM, Miller JH, Calos MP. Analysis of mutation in human cells by using an Epstein-Barr virus shuttle system. Mol Cell Biol 1987; 7: 379-387.

12 Ausubel $L$, Hall C, Sharma A, Shakeley R, Lopez P, Quezada V et al. Production of CGMP-grade lentiviral vectors. Bioprocess Int 2012; 10: 32-43.

13 Follenzi A, Naldini L. HIV-based vectors. Preparation and use. Methods Mol Med 2002; 69: 259-274.

14 Tiscornia G, Singer O, Verma IM. Production and purification of lentiviral vectors. Nat Protoc 2006; 1: 241-245.

15 Lesch HP, Heikkilä KM, Lipponen EM, Valonen P, Müller A, Räsänen E et al. Process development of adenoviral vector production in fixed bed bioreactor: from bench to commercial scale. Hum Gene Ther 2015; 26: 560-571.
16 Wang X, Olszewska M, Qu J, Wasielewska T, Bartido S, Hermetet G et al. Largescale clinical-grade retroviral vector production in a fixed-bed bioreactor. J Immunother 2015; 38: 127-135.

17 Ansorge S, Lanthier S, Transfiguracion J, Durocher Y, Henry O, Kamen A. Development of a scalable process for high-yield lentiviral vector production by transient transfection of HEK293 suspension cultures. J Gene Med 2009; 11: 868-876.

18 Kuroda H, Kutner RH, Bazan NG, Reiser J. Simplified lentivirus vector production in protein-free media using polyethylenimine-mediated transfection. J Virol Methods 2009; 157: 113-121.

19 Rodrigues AF, Carmo M, Alves PM, Coroadinha AS. Retroviral vector production under serum deprivation: the role of lipids. Biotechnol Bioeng 2009; 104: 1171-1181.

20 Lennaertz A, Knowles S, Drugmand J-C, Castillo J. Viral vector production in the integrity iCELLis" single-use fixed-bed bioreactor, from bench-scale to industrial scale. BMC Proc 2013; 7: P59.

21 Rajendran R, Lingala R, Vuppu SK, Bandi BO, Manickam E, Macherla SR et al. Assessment of packed bed bioreactor systems in the production of viral vaccines. AMB Express 2014; 4: 25.

22 Ventini-Monteiro D, Dubois S, Astray RM, Castillo J, Pereira CA. Insect cell entrapment, growth and recovering using a single-use fixed-bed bioreactor. Scaling up and recombinant protein production. J Biotechnol 2015; 216: 110-115.

23 Powers AD, Piras BA, Clark RK, Lockey TD, Meagher MM. Development and optimization of AAV hFIX particles by transient transfection in an iCELLis( $\left.{ }^{\circ}\right)$ fixedbed bioreactor. Hum Gene Ther Methods 2016; 27: 112-121.

24 Thomas P, Smart TG. HEK293 cell line: a vehicle for the expression of recombinant proteins. J Pharmacol Toxicol Methods 51: 187-200.

25 McCarron A, Donnelley M, McIntyre C, Parsons D. Challenges of up-scaling lentivirus production and processing. J Biotechnol 2016; 240: 23-30.

26 Holic N, Seye AK, Majdoul S, Martin S, Merten OW, Galy A et al. Influence of mildly acidic $\mathrm{pH}$ conditions on the production of lentiviral and retroviral vectors. Hum Gene Ther Clin Dev 2014; 25: 178-185.

27 Tang Y, Garson K, Li L, Vanderhyden BC. Optimization of lentiviral vector production using polyethylenimine-mediated transfection. Oncol Lett 2015; 9: $55-62$.

28 Karolewski BA, Watson DJ, Parente MK, Wolfe JH. Comparison of transfection conditions for a lentivirus vector produced in large volumes. Hum Gene Ther 2003; 14: 1287-1296.

29 Logan AC, Nightingale SJ, Haas DL, Cho GJ, Pepper KA, Kohn DB. Factors influencing the titer and infectivity of lentiviral vectors. Hum Gene Ther 2004; 15: 976-988.

30 Koponen JK, Kekarainen T, E Heinonen S, Laitinen A, Nystedt J, Laine J et al. Umbilical cord blood-derived progenitor cells enhance muscle regeneration in mouse hindlimb ischemia model. Mol Ther 2007; 15: 2172-2177.

31 Piatak M, Yang LC, Luk KC, Lifson JD, Saag MS, Clark SJ et al. Viral dynamics in primary HIV-1 infection. Lancet (London, England) 1993; 341: 1099.

32 Vogt VM. Retroviral virions and genomes 1997.

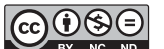

This work is licensed under a Creative Commons AttributionNonCommercial-NoDerivs 4.0 International License. The images or other third party material in this article are included in the article's Creative Commons license, unless indicated otherwise in the credit line; if the material is not included under the Creative Commons license, users will need to obtain permission from the license holder to reproduce the material. To view a copy of this license, visit http:// creativecommons.org/licenses/by-nc-nd/4.0/

(c) The Author(s) 2018

Supplementary Information accompanies this paper on Gene Therapy website (http://www.nature.com/gt) 